\title{
THE NATURE OF ULTRALUMINOUS IRAS GALAXIES
}

\author{
D.L. CLEMENTS \\ Institut d'Astrophysique Spatial \\ Bat 121, Universite Paris XI, \\ F-91405 ORSAY CEDEX, France \\ W.J. SUTHERLAND \\ Oxford University Astrophysics \\ NAPL, Keble Road, Oxford, UK \\ AND \\ R.G. MCMAHON \\ Institute of Astronomy \\ Madingley Road, Cambridge, UK
}

We present the results of a continuing programme to investigate the properties of the Ultraluminous IRAS Galaxies (ULIRGs) in a large sample selected from the IRAS Faint Source Catalogue and identified with the APM galaxy catalogue (for details of the survey selection and initial results see Clements et al. 1996, MNRAS, 279, 459). Observations now include optical imaging for a complete subsample to $B_{\mathrm{J}}=19.5$ (Clements et al. 1996, MNRAS, 279, 477) and near-IR imaging for a complete subsample to $B_{\mathrm{J}}=19$ (Clements et al. in preparation). Moderate resolution optical spectroscopy, ISOPHOT photometry and ISOSWS spectroscopy have also been obtained for smaller subsamples of this survey. New redshift measurements have identified 11 new ULIRGs, extending the sample size to 102 ULIRGs. These observations have extended the maximum redshift to 0.44 , and have also discovered a new broad-line ULIRG at $z=0.351$. Initial analysis of our ISOPHOT data, while subject to substantial calibration uncertainties, shows a broad range of $160-120 \mu \mathrm{m}$ flux ratios which might indicate the presence of cold dust $(T \sim 20 \mathrm{~K})$ in some objects. Such dust would not have been detectable by IRAS. Our continuing multiwavelength studies of ULIRGs will be used to examine the nature of the power source in these objects, and their relationship to other extragalactic populations. 